\title{
Communication
}

\section{Low-Frequency Content of THz Emission from Two-Color Femtosecond Filament}

\author{
Daniil E. Shipilo 1,*(D), Nikolay A. Panov ${ }^{1,2}$, Irina A. Nikolaeva ${ }^{1,2}$, Alexander A. Ushakov ${ }^{3}$ (D), \\ Pavel A. Chizhov ${ }^{3,4} \mathbb{D}$, Kseniia A. Mamaeva ${ }^{3}$, Vladimir V. Bukin ${ }^{3} \mathbb{D}$, Sergey V. Garnov ${ }^{3}$ and Olga G. Kosareva $^{1,2} \mathbb{D}^{\circ}$ \\ 1 P.N. Lebedev Physical Institute of the Russian Academy of Sciences, 119991 Moscow, Russia; \\ napanov@ilc.edu.ru (N.A.P.); nikolaevaia@lebedev.ru (I.A.N.); kosareva@physics.msu.ru (O.G.K.) \\ 2 Faculty of Physics, Lomonosov Moscow State University, 119991 Moscow, Russia \\ 3 Prokhorov General Physics Institute of the Russian Academy of Sciences, 119991 Moscow, Russia; \\ ushakov.aleksandr@physics.msu.ru (A.A.U.); pvch@kapella.gpi.ru (P.A.C.); \\ mamaeva@physics.msu.ru (K.A.M.); vbkn@kapella.gpi.ru (V.V.B.); garnov@kapella.gpi.ru (S.V.G.) \\ 4 Russian Institute for Scientific and Technical Information, 125315 Moscow, Russia \\ * Correspondence: schipilo.daniil@physics.msu.ru
}

check for

updates

Citation: Shipilo, D.E.; Panov, N.A.; Nikolaeva, I.A.; Ushakov, A.A.; Chizhov, P.A.; Mamaeva, K.A.; Bukin, V.V.; Garnov S.V.; Kosareva, O.G. Low-Frequency Content of $\mathrm{THz}$ Emission from Two-Color Femtosecond Filament. Photonics 2022, 9, 17. https://doi.org/ $10.3390 /$ photonics 9010017

Received: 23 November 2021 Accepted: 27 December 2021 Published: 29 December 2021

Publisher's Note: MDPI stays neutral with regard to jurisdictional claims in published maps and institutional affiliations.

Copyright: (C) 2021 by the authors. Licensee MDPI, Basel, Switzerland. This article is an open access article distributed under the terms and conditions of the Creative Commons Attribution (CC BY) license (https:// creativecommons.org/licenses/by/ $4.0 /)$.

\begin{abstract}
We experimentally investigate the low-frequency (below $1 \mathrm{THz}$ ) spectral content of broadband terahertz (THz) emission from two-color femtosecond filament formed by the 2.7-mJ, 40-fs, $800+400-n m$ pulse focused into air. For incoherent detection, we screened the Golay cell by the bandpass filters and measured the THz angular distributions at the selected frequencies $v=0.5,1,2$ and $3 \mathrm{THz}$. The measured distributions of $\mathrm{THz}$ fluence were integrated over the forward hemisphere taking into account the transmittance of the filters, thus providing the estimation of spectral power at the frequencies studied. The spectral power decreases monotonically with the frequency increasing from 0.5 to $3 \mathrm{THz}$, thus showing that the maximum of $\mathrm{THz}$ spectrum is attained at $v \leq 0.5 \mathrm{THz}$. The $\mathrm{THz}$ waveform measured by electro-optical sampling (EOS) based on ZnTe crystal and transformed into the spectral domain shows that there exists the local maximum of the $\mathrm{THz}$ spectral power at $v \approx 1 \mathrm{THz}$. This disagrees with monotonic decrease of $\mathrm{THz}$ spectral power obtained from the filter-based measurements. We have introduced the correction to the spectral power reconstructed from EOS measurements. This correction takes into account different focal spot size for different $\mathrm{THz}$ frequencies contained in the broadband electromagnetic pulse. The corrected EOS spectral power is in semi-quantitative agreement with the one measured by a set of filters.
\end{abstract}

Keywords: two-color femtosecond filament; terahertz radiation; low-frequency terahertz content

\section{Introduction}

Two-color femtosecond filament [1] with the intensity of $\sim 100 \mathrm{TW} / \mathrm{cm}^{2}$ [2-4], the beam diameter of 50-100 $\mu \mathrm{m}[5-7]$, and the plasma density of $10^{15}-10^{19} \mathrm{~cm}^{-3}[8,9]$ is a terahertz (THz) source with the most broadband spectrum up to $50-100 \mathrm{THz}$ [10-12], depending on the pump pulse duration [13]. The high-frequency THz emission from twocolor filament has been studied in details both experimentally and theoretically [13-17]. So, the angular distribution in the range of frequencies $v \gtrsim 5 \mathrm{THz}$ is conical $[16,18-21]$, but the shape of directional diagrams at lower frequencies is still debated [22]. Indeed, the experiments [21-23] show the unimodal on-axis angular distribution of $\mathrm{THz}$ emission from two-color filament for $v \lesssim 3 \mathrm{THz}$. However, in other experiments [24-26], the conical divergence of $\mathrm{THz}$ radiation was observed.

The spectral distribution of the low-frequency THz emission from a two-color filament is still unknown. Even the position of the low-frequency maximum $v_{\max }$ in the $\mathrm{THz}$ spectra measured in the close conditions (pulse energy and duration, geometry of the experiment etc.) can vary by an order of magnitude from one experiment to another. We collected the corresponding data in Table 1, which shows that as the pump pulse duration varies in the range of 30-50 fs, the position of the spectral maximum travels in a wide frequency 
range from $0.4 \mathrm{THz}$ [27] to $13 \mathrm{THz}$ [28]. Self-consistent numerical simulations based on Unidirectional pulse propagation Equation (UPPE [29]) predict the maximum of $\mathrm{THz}$ spectrum at the frequency of about a few hundreds of gigahertz $[16,17]$.

Table 1. The frequency of maximum $v_{\max }$ in the $\mathrm{THz}$ spectra measured in different experiments in the conditions close to the ones of our experiment.

\begin{tabular}{|c|c|c|c|c|c|}
\hline $\begin{array}{c}\text { Pulse } \\
\text { Duration, fs }\end{array}$ & $\begin{array}{c}\text { Pulse } \\
\text { Energy, } \mathrm{mJ}\end{array}$ & $\begin{array}{l}\text { Focusing } \\
\text { Conditions }\end{array}$ & $\begin{array}{l}\text { Detection } \\
\text { Method }\end{array}$ & $v_{\max }, \mathrm{THz}$ & Ref. \\
\hline 32 & $\sim 1$ & $f=15 \mathrm{~cm}$ & EOS & 2.9 & [13] \\
\hline 32 & 0.7 & $f=15 \mathrm{~cm}$ & $\mathrm{ABCD}$ & 4.4 & [30] \\
\hline 32 & 0.7 & - & $\mathrm{ABCD}$ & $\sim 5$ & [31] \\
\hline 35 & 2 & $f=25 \mathrm{~cm}$ & EOS & 0.4 & [27] \\
\hline 35 & $\sim 3$ & $f / 15$ & Interferometer & 13 & [28] \\
\hline 40 & $0.7-2$ & $f=15-25 \mathrm{~cm}$ & EOS & $\sim 1$ & [32] \\
\hline 40 & $\sim 1$ & $f=15 \mathrm{~cm}$ & EOS & 2.3 & [13] \\
\hline 40 & 2.3 & $f=20 \mathrm{~cm}$ & $\begin{array}{c}\text { EOS (ZnTe) } \\
\text { EOS (GaP) } \\
\text { ABCD } \\
\text { Interferometer }\end{array}$ & $\begin{array}{c}1.7 \\
2.2 \\
3.4 \\
7\end{array}$ & [33] \\
\hline 40 & $1-6$ & $f / 10$ & Interferometer & $5-10$ & [34] \\
\hline 50 & 0.5 & $f=30 \mathrm{~cm}$ & EOS & 0.4 & [35] \\
\hline 50 & 1.15 & $f / 80$ & EOS & $\sim 0.5$ & [36] \\
\hline 50 & 2.5 & $f / 40-f / 4$ & EOS & $0.5-0.7$ & [37] \\
\hline 50 & 1.7 & $f=20 \mathrm{~cm}$ & EOS & $\sim 1$ & [38] \\
\hline 50 & $\sim 1$ & - & $\begin{array}{l}\text { ABCD } \\
\text { EOS }\end{array}$ & $\begin{array}{c}0.55 \\
1.7\end{array}$ & [31] \\
\hline
\end{tabular}

This large spreading of experimentally obtained frequencies $v_{\text {max }}$ can be attributed to the extreme broadness of the spectrum of $\mathrm{THz}$ emission from two-color filament. Different components of the THz spectrum have different divergence diagrams. As the result, the $\mathrm{THz}$ focal spot size varies significantly across the spectrum. This focal spot size variation affects the collection of $\mathrm{THz}$ radiation by coherent detection schemes, in particular, electrooptical sampling EOS [39] and air-biased coherent detection ABCD [40]. Both methods require overlap between the probe optical pulse and the focused $\mathrm{THz}$ pulse. The $\mathrm{THz}$ spot transverse size is of order of $\mathrm{THz}$ wavelength or larger, and, therefore, exceeds the typical $\sim 1 \mathrm{~mm}$ (or even less in case of ABCD detection) diameter of the probe beam [41,42]. For example, at the low $\mathrm{THz}$ frequency $v \lesssim 0.5 \mathrm{THz}$ with the corresponding wavelength larger than $\sim 600 \mu \mathrm{m}$, the THz beam size in the focus is equal or larger than $1 \mathrm{~mm}$. As a result, a significant fraction of the $\mathrm{THz}$ beam does not overlap with the probe, and the coherent time domain sampling (TDS) underestimates the low-frequency THz content [43].

It should be noted that this underestimation does not prevent the time-domain sampling from being an excellent tool in THz spectroscopy [44]. Indeed, through the comparison of the spectrum of $\mathrm{THz}$ radiation transmitted through the substance and the one of the reference THz signal, TDS provides the information about both real and imaginary parts of the dielectric susceptibility [39]. The low in the amplitude half-cycle electric field in a standard EOS crystal ZnTe $(89 \mathrm{kV} / \mathrm{cm}$ for a $1 \mathrm{~mm}$ thickness [45]) makes the registration of a weak THz signal possible. Using EOS, Zhong et al. recorded the THz spectra of explosives from the distance of $30 \mathrm{~m}$ in the atmosphere [46]. The EOS scheme can be adapted for $2 D$ frequency-resolved imaging of organic substances [47]. The ABCD technique was applied 
to broadband THz spectroscopy $[48,49]$. The dispersion of TDS sensitivity becomes a problem only if one decides to characterize the $\mathrm{THz}$ source rather than the sample.

The incoherent methods of $\mathrm{THz}$ spectra registration based on the Michelson interferometer coupled to a $\mathrm{THz}$ powermeter (a bolometer or a Golay cell) are aimed at the high-frequency $\mathrm{THz}$ content [33]. The THz spectra measured by these devices have the maximum at high $\mathrm{THz}$ frequencies $v_{\max } \geq 5 \mathrm{THz}$, see Table 1 . The strongly divergent lowfrequency $\mathrm{THz}$ radiation diffracts out of the aperture of the interferometer mirrors and does not enter the powermeter. Therefore, the low-frequency $\mathrm{THz}$ content is underestimated in such interferometric measurements as well.

The purpose of this work is to study experimentally the low-frequency $(v \lesssim 1 \mathrm{THz})$ spectral content of the broadband $\mathrm{THz}$ emission from two-color femtosecond filament, formed in air by $2.7-\mathrm{mJ}, 40-\mathrm{fs}, 800+400-\mathrm{nm}$ pulse in the vicinity of $20-\mathrm{cm}$ geometrical focus. To reconstruct the spectral components of $\mathrm{THz}$ emission from two-color filament, we measured the angular distributions of the $\mathrm{THz}$ radiation by rotation of the Golay cell, the entrance window of which was screened by the bandpass filters centered at $0.5,1,2$ and $3 \mathrm{THz}$ (see Figure 1a). The spectrum reconstructed from $\mathrm{THz}$ angular distributions was compared with the one measured by electro-optical sampling for the same two-color filament (see Figure 1b). The comparison shows relatively low power at the frequency below $1 \mathrm{THz}$ in the case of EOS measurement. We found that the qualitative agreement between the two spectra is achieved if the spectrum obtained from EOS is corrected based on the convolution between the optical probe and THz pump pulse in ZnTe crystal.

(a)

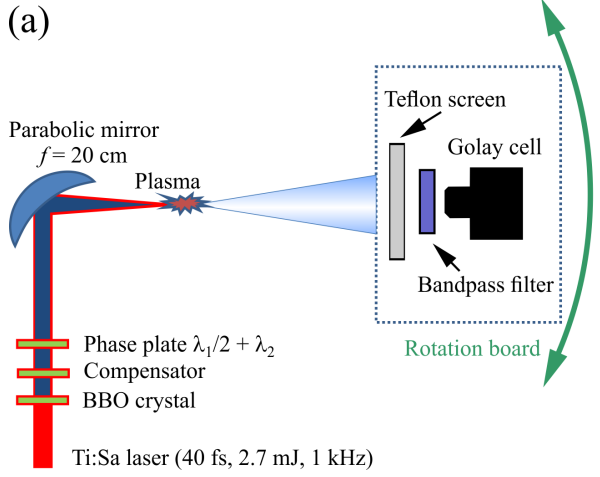

(b)

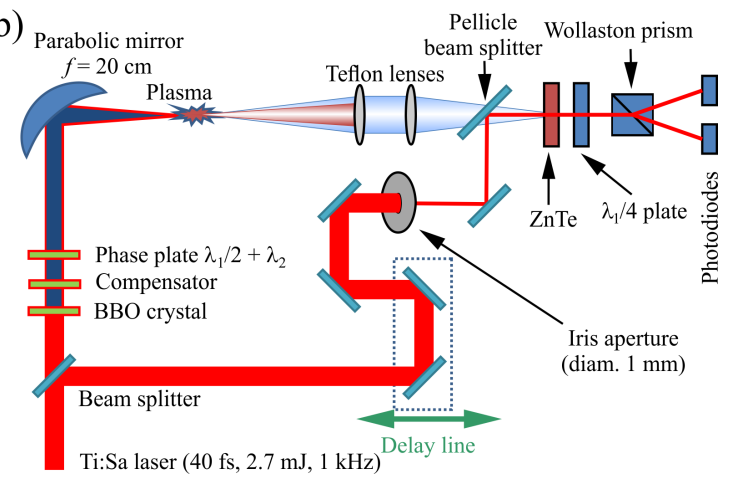

Figure 1. Experimental schemes. (a) Angular distribution measurements. Golay cell with a bandpass filter and teflon screen is mounted on the board rotating around the geometrical focus along the green arc with arrows on both sides. (b) Time-domain measurements. A focused THz beam is combined on the $\mathrm{ZnTe}$ crystal with a probe pulse, the time delay of which is varied by moving the delay line along the green line with arrows on both sides.

\section{Materials and Methods}

In the experiment we used the laser pulses delivered by Ti:Sapphire laser system Spectra Physics Spitfire Pro XP centred at the wavelength $\lambda_{1}=800 \mathrm{~nm}$ with the energy up to $2.7 \mathrm{~mJ}$, FWHM duration of $40 \mathrm{fs}$ and beam diameter of $12 \mathrm{~mm}$ (at $e^{-2}$ level) at $1 \mathrm{kHz}$ repetition rate. To form the two-color filament we inserted a BBO crystal (I-type, $100-\mu \mathrm{m}$ thickness, $10 \%$ efficiency) into the pump beam thus providing generation of the second harmonic radiation centered at $\lambda_{2}=400 \mathrm{~nm}$ (Figure 1). The BBO crystal was adjusted to maximize the second harmonic yield. Behind the $\mathrm{BBO}$ crystal, polarization of the 400-nm radiation was oriented perpendicularly to the one of the $800-\mathrm{nm}$ pump. Similarly to our work [23], a compensator plate decreased the group delay between the fundamental $\left(\lambda_{1}\right)$ and the second $\left(\lambda_{2}\right)$ harmonic pulses. A dual wavelength phase plate (" $\lambda_{1} / 2+\lambda_{2}$ " in Figure 1) served to align the polarization vectors of the harmonic pulses in order to maximize the THz yield. Thereby, both harmonics had the same vertical linear polarization direction and zero group delay between themselves in the filament. After the dual wavelength phase plate the two-color beam was focused into the atmospheric 
air by the off-axis parabolic mirror with the focal length $f=20 \mathrm{~cm}$. In the vicinity of the geometrical focus, the $\sim 10-\mathrm{mm}$ two-color filament was formed. It was the source of $\mathrm{THz}$ radiation, which spectral content was studied in our experiment.

We measured the spectral content of $\mathrm{THz}$ emission from two-color laser-induced plasma using two different techniques. In the first case (Figure 1a), we screened the Golay cell by the teflon plate (to block laser radiation) and the bandpass filters (Tydex [50]) centered at the frequencies $v=0.5,1,2$ and $3 \mathrm{THz}$. The transmittance spectra provided by the manufacturer for our filters are shown in Figure 2 by the colored areas. We rotated the Golay cell placed at a distance of $20 \mathrm{~cm}$ from the geometrical focus around the vertical axis by the angle $\theta$ relative to the laser beam axis with the step $\Delta \theta=2.5^{\circ}$ (Figure 1a). With this setup we recorded the angular distributions of $\mathrm{THz}$ fluence $F(\theta)$ at different frequencies $v$. In these measurements the Pockels cell located in the regenerative amplifier provided the modulation of generated $\mathrm{THz}$ emission with a frequency of $20 \mathrm{~Hz}$. The resulting $\mathrm{THz}$ signal was registered by means of synchronous detection technique using lock-in amplifier. The measured dependencies $F(\theta)$ were processed according to Ref. [51] to obtain the power $S$ of the spectral components at the set of frequencies studied.

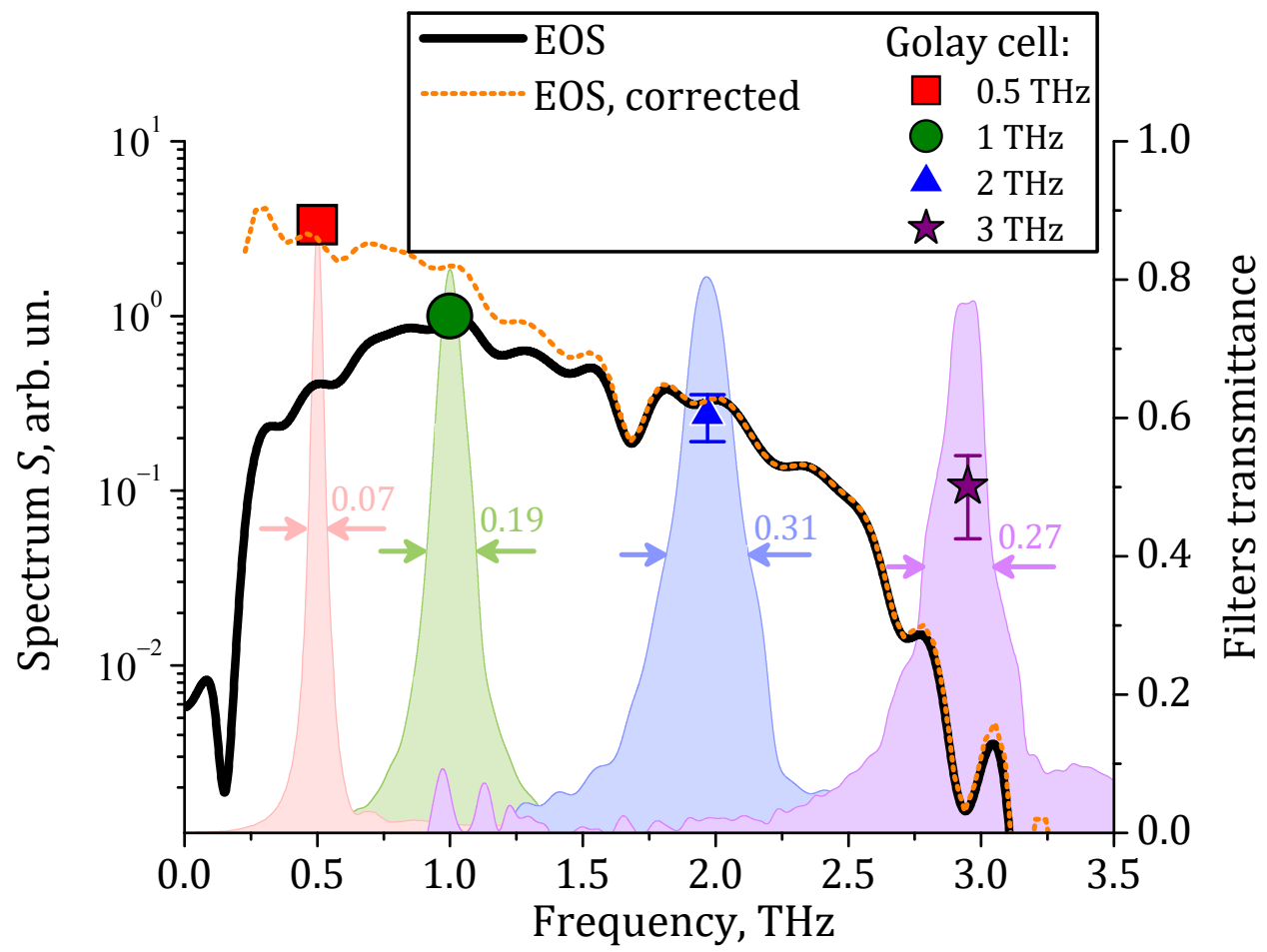

Figure 2. Spectrum of $\mathrm{THz}$ radiation obtained after integration of measured by the Golay cell with bandpass filters angular distributions of THz spectral intensity $I(\theta)$ (symbols, left axis) and spectrum measured by EOS (black curve, left axis). The orange dotted curve (left axis) is the spectrum calculated from the one measured by EOS (the black solid curve) using the EOS sensitivity from Appendix A, Equation (A11). The colored areas (right axis) show $\mathrm{THz}$ bandpass filters transmittance spectra. Arrows with numbers indicate their full width at half maximum $\Delta v$ in terahertz.

In the second case (Figure $1 \mathrm{~b}$ ), we measured $\mathrm{THz}$ waveforms using a standard timedomain system based on the electro-optics sampling technique (EOS [39]). The system consisted of the ZnTe crystal $\left(10 \times 10 \times 0.5 \mathrm{~mm}^{3},\langle 110\rangle\right.$ cut), the quarterwave plate (" $\lambda_{1} / 4$ " in Figure 1b), the Wollaston prism, and two photodiodes. This setup registered the radiation in the spectral range from $\sim 0.2$ to $\sim 3 \mathrm{THz}$. Two $50-\mathrm{mm}$ in diameter teflon lenses with the foci of 6 and $10 \mathrm{~cm}$ collimated the THz radiation and focused it onto the ZnTe crystal, respectively (Figure 1b). Optical probe pulse passed through a variable delay line and then was combined with the $\mathrm{THz}$ pulse on a lavsan pellicle. So as to decrease a fluctuation 
of registered signal, we apertured the collimated probe beam down to $1 \mathrm{~mm}$ in diameter. By the variation of the delay between the probe and $\mathrm{THz}$ pulses, we measured the $\mathrm{THz}$ waveforms. The $\mathrm{THz}$ spectrum was reconstructed from the measured waveform using the Fourier transform.

\section{Results and Discussion}

Figure 3a shows the dependence of the $\mathrm{THz}$ fluence $F$ on the polar angle $\theta$ measured by the Golay cell at the frequency $v=0.5 \mathrm{THz}$ (red squares), $1 \mathrm{THz}$ (green circles), $2 \mathrm{THz}$ (blue triangles) and $3 \mathrm{THz}$ (violet stars). Angular distributions $F(\theta)$ get narrower with the frequency increase in reasonable agreement with the well-known law [52] for the divergence angle

$$
\delta \propto \sqrt{v^{-1}}
$$

of electromagnetic waves emitted from an extended source, see Figure 4. Equation (1) is usually used in the studies of $\mathrm{THz}$ emission from a single-color filament [53-55], however, is valid in the case of two-color filament [18], and even for wire lasers [56].
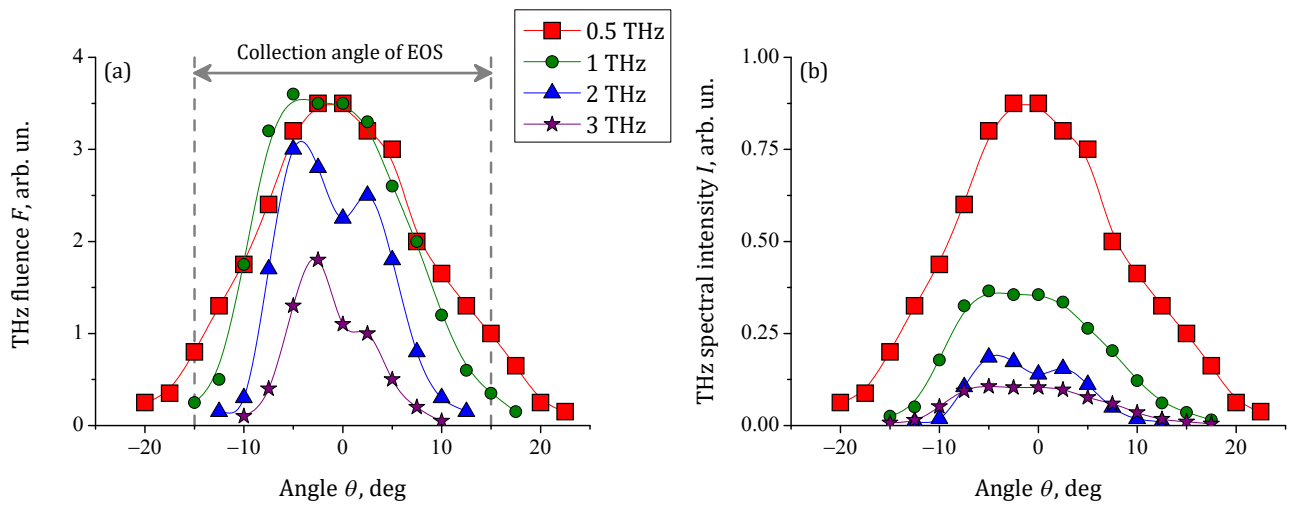

Figure 3. Angular distributions of (a) THz fluence $F(\theta)$ measured by the Golay cell with bandpass filters at the frequencies $v=0.5 \mathrm{THz}$ (red squares), $1 \mathrm{THz}$ (green circles), $2 \mathrm{THz}$ (blue triangles), $3 \mathrm{THz}$ (violet stars) and (b) $\mathrm{THz}$ spectral intensity $I(\theta)$ obtained from (a) by the correction to the maximal transmittance of the filters $T$ and their bandwidth $\Delta v$, see Equation (2).

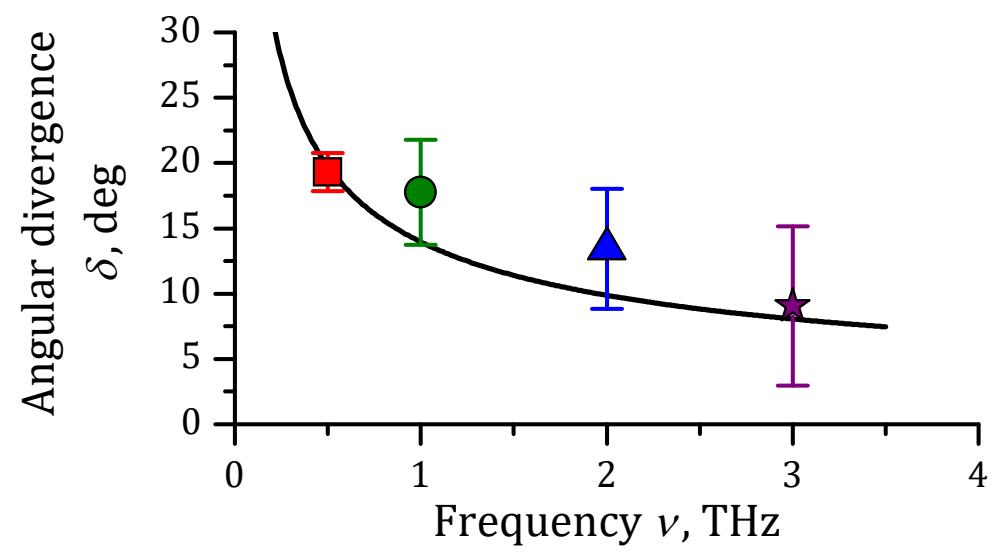

Figure 4. Dependence of the angular divergence $\delta$ of THz radiation on frequency $v$ estimated from angular distributions of $\mathrm{THz}$ fluence $F(\theta)$ (symbols). Black solid curve shows the fit of the angular divergence by Equation (1). Symbols correspond to the ones in Figure 3.

Bandpass filters centered at different frequencies $v$ have different transmittance spectra, see colored areas in Figure 2. So, to compare the amplitudes of $\mathrm{THz}$ signal measured with 
different filters, we recalculated the measured $\mathrm{THz}$ fluence $F$ (see Figure 3a) into the $\mathrm{THz}$ spectral intensity $I$ (see Figure $3 b$ ) according to the equation:

$$
I(\nu, \theta)=\frac{F(\nu, \theta)}{T(v) \Delta v(v)} .
$$

Equation (2) takes into account the transmittance of the filters $T$, their bandwidth $\Delta v$ (see colored areas in Figure 2) and the constant spectral sensitivity of the Golay cell within the range of frequency studied, that is from 0.5 to $3 \mathrm{THz}$. The maximal transmittance $T$ slightly changes with the central frequency of the bandpass filter $v$. At the same time, the filter bandwidth $\Delta v$ increases fourfold with the central frequency $v$ increase from 0.5 to $3 \mathrm{THz}$ (cf. red and violet colored areas in Figure 2). This results in monotonic decrease in the angularly-resolved THz spectral intensity I with the frequency increase (Figure $3 \mathrm{~b}$ ). In contrast, the measured $\mathrm{THz}$ fluence profiles $F$ have almost the same amplitude for all the frequencies studied (Figure 3a).

According to 2D measurements of the THz transverse spatial distribution $[18,19,57]$, the latter is axially symmetrical in the case of geometrical focusing into air of the moderatepower pulse [58]. Assuming axial symmetry of $\mathrm{THz}$ beam in our experiments, we integrate the directional diagrams $I(v, \theta)$ over the whole forward hemisphere to obtain the spectral power of $\mathrm{THz}$ radiation $S(v)$ :

$$
S(v)=\Delta \theta \sum_{p} I(v, p \Delta \theta)|\sin (p \Delta \theta)|
$$

where $\Delta \theta=2.5^{\circ}$ and the integer index $p$ runs over the angular positions of the Golay cell. The spectral powers $S(v)$ calculated from Equation (3) and divided by $S(v=1 \mathrm{THz})$ are shown in Figure 2 by symbols corresponding to the ones in Figure 3. The uncertainties are estimated from the asymmetry of $\mathrm{THz}$ divergence diagrams, see Figure 3 . The dependence $S(v)$ decreases monotonically as the frequency $v$ grows. Therefore, the frequency $v_{\max }$ of the maximum in $\mathrm{THz}$ spectrum is below $0.5 \mathrm{THz}$.

We compare the spectral power $S$ estimated from Equation (3) with the spectrum measured by EOS and shown in Figure 2 by the black curve. Both spectra are normalized to the spectral power at $v=1 \mathrm{THz}$, at which the spectral maximum in the EOS measurement is achieved. In the range of frequencies $v=1-2 \mathrm{THz}$ the spectral powers obtained by the two methods coincide within an error. At the highest frequency $v=3 \mathrm{THz}$, the spectral power reconstructed from $\mathrm{THz}$ angular distribution exceeds the one measured by EOS by approximately two orders of magnitude due to ZnTe phonon band at $v \gtrsim 3 \mathrm{THz}$ [59].

However, the spectral power measured by EOS at $v=0.5 \mathrm{THz}$ is an order of magnitude lower than the one obtained from integration of the angular distribution (compare the black curve at $v=0.5 \mathrm{THz}$ with the red square in Figure 2). The overall collection angle of the $\mathrm{THz}$ collimatimg lens (Figure $1 \mathrm{~b}$ ) is about $30^{\circ}$. Therefore, only a little portion of $\mathrm{THz}$ emission from the two-color filament escapes the lens and, hence, the ZnTe crystal (Figure 3a). The depletion at $v=0.5 \mathrm{THz}$ in the THz spectrum measured by EOS can be formed due to geometrical focusing of $\mathrm{THz}$ radiation into the spot larger than the probe beam transverse size. We developed a model of EOS detection technique, which includes the effect of the broadband THz geometrical focusing onto the ZnTe crystal, see Appendix A. Figure 5a shows the EOS spectral sensitivity $\varepsilon(v)$, calculated according to this model in our experimental conditions, i.e., for the probe beam, which was apertured down to $1 \mathrm{~mm}$ and afterwards propagated for about $50 \mathrm{~cm}$ towards the ZnTe crystal. The diffraction of the apertured probe beam forms the $\sim 400-\mu \mathrm{m}$ in diameter quasi-Gaussian beamlet surrounded by divergent rings (Figure $5 b, c)$. We corrected the measured by EOS spectrum taking into account the EOS sensitivity $\varepsilon(v)$, see Figure 2, dotted curve. The depletion in the corrected EOS spectrum vanished at $v<1 \mathrm{THz}$ in agreement with the spectral power $S$ at $v=0.5 \mathrm{THz}$ (Figure 2, compare black solid and orange dotted curves). This agreement between the corrected EOS spectrum and the spectrum obtained by the integration over the forward hemisphere confirms our result, that the actual spectrum of 
THz emission from two-color filament has the maximum at few hundreds of gigahertz or even less.
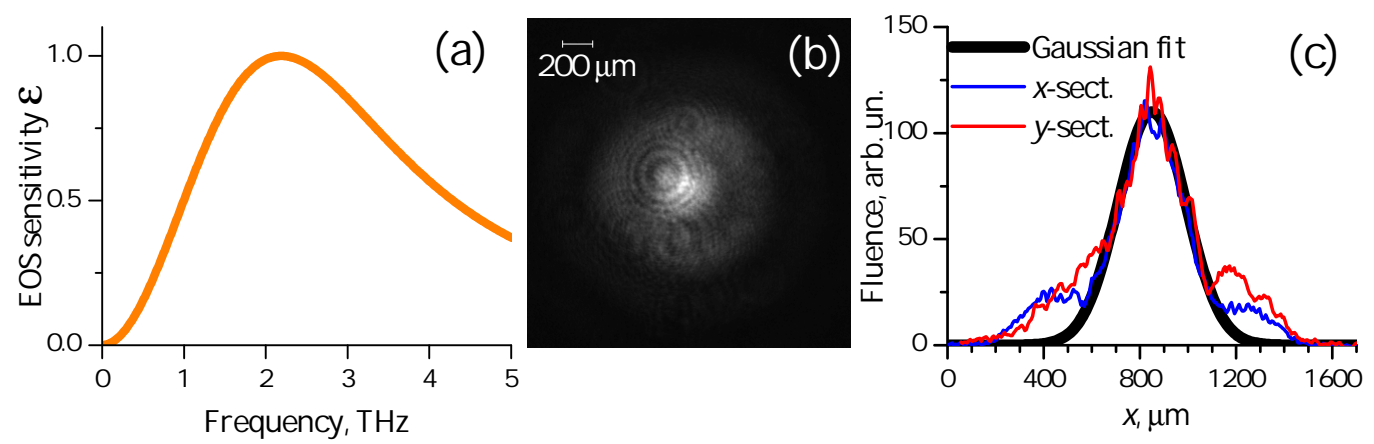

Figure 5. (a) Sensitivity of EOS calculated from Equation (A11) assuming Gaussian spatial distribution of the probe beam, $\exp \left[-r^{2} /(200 \mu \mathrm{m})^{2}\right]$. (b) Spatial fluence distribution of the probe beam on the ZnTe crystal measured by CCD. (c) Cross-sections of the probe beam and the Gaussian fit of its most-intense part.

\section{Conclusions}

In conclusion, the incoherent measurements of the frequency-angular distribution of $\mathrm{THz}$ emission from two-color femtosecond filament indicate the monotonic decrease in spectral power from 0.5 to $3 \mathrm{THz}$ and, thus, the absence of spectral maximum in this range. The measured by the rotation of the Golay cell screened by the bandpass filters $\mathrm{THz}$ angular distributions were integrated over the forward hemisphere taking into account the filters' transmittance. As a result, we reconstructed the spectral powers at the filters' central frequencies. Our technique of the incoherent measurements of $\mathrm{THz}$ spectrum does not use the focusing/collimating THz elements and, therefore, allows us to estimate the actual low-frequency content of $\mathrm{THz}$ emission from a two-color filament.

In contrast, the spectrum obtained by electro-optical sampling in the same conditions has a maximum at $1 \mathrm{THz}$. The depletion of low-frequency components in EOS measurements is due to their geometrical focusing into the spot larger than the probe beam diameter and, therefore, weakening of the $\mathrm{THz}$-induced birefrigence of the probe beam. The model of EOS, which accounts for the nonparaxial diffraction and focusing of $\mathrm{THz}$ wave, provides a correction factor to EOS spectrum. The corrected spectrum complies with the incoherent measurements.

Author Contributions: A.A.U., P.A.C., K.A.M. and V.V.B. performed the experiment; D.E.S., N.A.P., I.A.N. and O.G.K. performed the data analysis and developed the model of EOS; V.V.B., S.V.G. and O.G.K. supervized the whole study. All authors have read and agreed to the published version of the manuscript.

Funding: This work was supported by the Ministry of Science and Higher Education of the Russian Federation (project 075-15-2020-790).

Institutional Review Board Statement: Not applicable.

Informed Consent Statement: Not applicable.

Data Availability Statement: Data underlying the results presented in this paper are not publicly available at this time but may be obtained from the authors upon reasonable request.

Conflicts of Interest: The authors declare no conflict of interest. 


\section{Appendix A. Derivation of EOS Sensitivity}

In this Appendix, we derive the equation for EOS spectral sensitivity following from a finite collection aperture for $\mathrm{THz}$ wave and finite transverse and temporal extension of the probe pulse. Here we analyze the $\mathrm{THz}$ radiation within the $\mathrm{ZnTe}$ transparency window $0.2 \mathrm{THz}<v<3 \mathrm{THz}$. The iterative method of high-frequency $(v>3 \mathrm{THz})$ spectrum reconstruction from EOS measurements was proposed in Ref. [33].

Let $t$ be the time, $z$ the propagation coordinate, $x$ and $y$ the transverse coordinates, $r=\sqrt{x^{2}+y^{2}}$. The electro-optical crystal was $\langle 110\rangle$ cut, so its main axes are

$$
\vec{\xi}=\vec{x}, \quad \vec{\zeta}=\frac{\vec{y}+\vec{z}}{\sqrt{2}}, \quad \vec{\eta}=\frac{\vec{y}-\vec{z}}{\sqrt{2}} .
$$

The cubic crystals like $\mathrm{ZnTe}, \mathrm{GaP}$ etc. have only six non-zero components of the second-order susceptibility tensor: $\chi_{\xi \zeta \eta}^{(2)}=\chi^{(2)}$ with all possible permutations of indices. Let the incident optical and terahertz fields have only $y$-components, $L_{y}(t, r)$ and $T_{y}(t, r)$, respectively. In this case, the second-order polarization has only $x$-component, which value at the optical frequency is

$$
P_{x}=2 \chi^{(2)} L_{y} T_{y} \text {. }
$$

We neglect the linear susceptibility of the crystal and assume it to be thin enough for one-step solution of the propagation equation

$$
2 i k_{L} \frac{\partial L_{\alpha}}{\partial z}=-4 \pi k_{L}^{2} P_{\alpha}
$$

where $\alpha$ is either $x$ or $y, k_{L}$ is the wavenumber of optical pulse, so that

$$
\left(\begin{array}{l}
L_{x} \\
L_{y}
\end{array}\right)^{(\text {out })}=\left(\begin{array}{c}
L_{x} \\
L_{y}
\end{array}\right)^{(\text {in })}+2 \pi i k_{L} \Delta z\left(\begin{array}{c}
P_{x} \\
P_{y}
\end{array}\right)=\left(\begin{array}{c}
i \varkappa T_{y} \\
1
\end{array}\right) L_{y}^{(i n)}
$$

where $\Delta z$ is the crystal length, $\varkappa=4 \pi k_{L} \Delta z \chi^{(2)}$.

After the EO crystal, $\lambda / 4$ plate is applied:

$$
\left(\begin{array}{l}
L_{x} \\
L_{y}
\end{array}\right)^{(\lambda / 4)}=\frac{1}{2}\left(\begin{array}{cc}
1+i & 1-i \\
1-i & 1+i
\end{array}\right)\left(\begin{array}{l}
L_{x} \\
L_{y}
\end{array}\right)^{(\text {out })}
$$

Then the Wollaston prism separates the polarizations and direct two beams on the balance detector, which signal is

$$
S=\int\left(\left|L_{x}^{(\lambda / 4)}\right|^{2}-\left|L_{y}^{(\lambda / 4)}\right|^{2}\right) d t d x d y=-2 \varkappa \int T_{y}\left|L_{y}^{(i n)}\right|^{2} d t d x d y .
$$

Let us now assume that plane $\mathrm{THz}$ wave with temporal distribution $T^{(i n)}(t)$ [and spectrum $\hat{T}^{(i n)}(\omega)$ ] is focused by an ideal parabolic mirror. The distribution of $\mathrm{THz}$ field on the crystal displaced by $z$ from the focus of parabolic mirror can be described by Equation (36) from [60]. We take only scalar axially symmetric part of this non-paraxial solution (the paraxial approach to broadband $\mathrm{THz}$ focusing was developed earlier in [61]):

$$
\hat{T}_{y}(\omega, r, z)=-\frac{k}{4} \hat{T}^{(i n)}(\omega) \int_{0}^{\operatorname{asin} N A} J_{0}\left(k r \sin \theta_{s}\right) \exp \left(-i k z \cos \theta_{s}\right) \sin \theta_{s} d \theta_{s}
$$

where $k=\omega / c, N A$ is a numerical aperture of the parabola, $J_{0}$ is the zeroth order Bessel function, the geometrical focus corresponds to $r=0, z=0$. This field is now convoluted with the probe beam $\left|L_{y}^{(i n)}(t, r)\right|^{2}=L_{1}\left(t-t_{d}\right) L_{2}(r)$ where the THz-to-probe delay $t_{d}$ becomes the only argument of the balance detector signal $S$. 
The Fourier transform of $S\left(t_{d}\right)$ provides the spectrum of $\mathrm{THz}$ wave:

$$
\hat{S}\left(\omega_{d}\right) \propto \int d t_{d} \exp \left(-i \omega_{d} t_{d}\right) \int d t r d r L_{1}\left(t-t_{d}\right) L_{2}(r) \int d \omega \exp (i \omega t) \hat{T}_{y}(\omega, r)
$$

The convolutions in time yield a factor $\hat{L}_{1}(\omega) \delta\left(\omega-\omega_{d}\right)$, so the index in $\omega_{d}$ can be omitted. To eliminate the spatial convolution we introduce Equation (A7) with the substitution $\sigma=\sin \theta_{s}$ :

$$
\hat{S}(\omega) \propto k \hat{T}^{(i n)}(\omega) \hat{L}_{1}(\omega) \int r d r L_{2}(r) \int_{0}^{N A} \frac{\sigma d \sigma}{\sqrt{1-\sigma^{2}}} J_{0}(k r \sigma) \exp \left(-i k z \sqrt{1-\sigma^{2}}\right) .
$$

The integral over $r$ is the Hankel transform of $L_{2}$, so the final equation reads:

$$
\hat{S}(\omega) \propto \omega \hat{T}^{(i n)}(\omega) \hat{L}_{1}(\omega) \int_{0}^{N A} \frac{\sigma d \sigma}{\sqrt{1-\sigma^{2}}} \hat{L}_{2}(\sigma \omega / c) \exp \left(-i z \sqrt{1-\sigma^{2}} \omega / c\right)
$$

The spectral sensitivity $\varepsilon(\omega)=\left|\hat{S}(\omega) / \hat{T}^{(i n)}(\omega)\right|^{2}$ can be obtained numerically provided we have the measured spectrum and transverse distribution of the probe.

For the sake of analytical solution, we assume that the crystal is placed exactly in the geometrical focus $(z=0)$, and the probe beam is Gaussian $\left|L_{y}^{(i n)}(t, r)\right|^{2}=$ $\exp \left(-\left(t-t_{d}\right)^{2} / \tau_{0}^{2}-r^{2} / a_{0}^{2}\right)$. Then the Fourier and Hankel transforms as well as the integration are explicit:

$$
\frac{\hat{S}(\omega)}{\hat{T}^{(i n)}(\omega)} \propto\left[\operatorname{erfi} \varphi-\operatorname{erfi}\left(\varphi \sqrt{1-N A^{2}}\right)\right] \exp \left[-\frac{1}{4}\left(\omega \tau_{0}\right)^{2}-\varphi^{2}\right]
$$

with $\varphi=\omega a_{0} /(2 c)$.

If the crystal cannot be assumed to be thin, the propagation in the medium should be considered carefully [61] and Equation (A6) will be modified to

$$
S \propto \int T_{y}(t, r, z)\left|L_{y}^{(i n)}(t, r, z)\right|^{2} d t d x d y d z
$$

with additional integration over the crystal length. The solution given by Equation (A12) can be analyzed numerically after substitution of Equation (A7). However, such solution does not have a closed form and depends on too many particular parameters of the experimental setup. This numerical approach makes sense only if one decides to improve the correction for higher $\mathrm{THz}$ frequencies, at which the beam distribution varies along the crystal length noticeably.

\section{References}

1. Cook, D.; Hochstrasser, R. Intense terahertz pulses by four-wave rectification in air. Opt. Lett. 2000, 25, 1210-1212. [CrossRef] [PubMed]

2. Kasparian, J.; Sauerbrey, R.; Chin, S.L. The critical laser intensity of self-guided light filaments in air. Appl. Phys. B 2000, 71, 877. [CrossRef]

3. Kosareva, O.G.; Liu, W.; Panov, N.A.; Bernhardt, J.; Ji, Z.; Sharifi, M.; Li, R.; Xu, Z.; Liu, J.; Wang, Z.; et al. Can we reach very high intensity in air with femtosecond PW laser pulses? Laser Phys. 2009, 19, 1776-1792. [CrossRef]

4. Mitryukovskiy, S.I.; Liu, Y.; Houard, A.; Mysyrowicz, A. Re-evaluation of the peak intensity inside a femtosecond laser filament in air. J. Phys. B 2015, 48, 094003. [CrossRef]

5. Braun, A.; Korn, G.; Liu, X.; Du, D.; Squier, J.; Mourou, G. Self-channeling of high-peak-power femtosecond laser-pulses in air. Opt. Lett. 1995, 20, 73. [CrossRef]

6. Pushkarev, D.; Mitina, E.; Shipilo, D.; Panov, N.; Uryupina, D.; Ushakov, A.; Volkov, R.; Karabutov, A.; Babushkin, I.; Demircan, A.; et al. Transverse structure and energy deposition by a subTW femtosecond laser in air: From single filament to superfilament. New J. Phys. 2019, 21, 033027. [CrossRef] 
7. Apeksimov, D.; Bukin, O.; Bykova, E.; Geints, Y.E.; Golik, S.; Zemlyanov, A.; Il'in, A.; Kabanov, A.; Matvienko, G.; Oshlakov, V.; et al. Filamentation of the focused Ti: Sapphire laser pulse in air at two harmonics. Plasma Phys. Rep. 2013, 39, $1074-1081$. [CrossRef]

8. Théberge, F.; Liu, W.; Simard, P.T.; Becker, A.; Chin, S.L. Plasma density inside a femtosecond laser filament in air: Strong dependence on external focusing. Phys. Rev. E 2006, 74, 036406. [CrossRef]

9. Ionin, A.A.; Kudryashov, S.I.; Seleznev, L.V.; Sinitsyn, D.V. Tunneling Ionization of Air in the Strong Field of Femtosecond Laser Pulses. JETP Lett. 2009, 90, 181-185. [CrossRef]

10. Fuji, T.; Suzuki, T. Generation of sub-two-cycle mid-infrared pulses by four-wave mixing through filamentation in air. Opt. Lett. 2007, 32, 3330-3332. [CrossRef]

11. Thomson, M.D.; Blank, V.; Roskos, H.G. Terahertz white-light pulses from an air plasma photo-induced by incommensurate two-color optical fields. Opt. Express 2010, 18, 23173-23182. [CrossRef]

12. Matsubara, E.; Nagai, M.; Ashida, M. Coherent infrared spectroscopy system from terahertz to near infrared using air plasma produced by 10-fs pulses. J. Opt. Soc. Am. B 2013, 30, 1627-1630. [CrossRef]

13. Borodin, A.V.; Panov, N.A.; Kosareva, O.G.; Andreeva, V.A.; Esaulkov, M.N.; Makarov, V.A.; Shkurinov, A.P.; Chin, S.L.; Zhang, X.C. Transformation of terahertz spectra emitted from dual-frequency femtosecond pulse interaction in gases. Opt. Lett. 2013, 38, 1906. [CrossRef]

14. Oh, T.; You, Y.; Jhajj, N.; Rosenthal, E.; Milchberg, H.; Kim, K.Y. Intense terahertz generation in two-color laser filamentation: Energy scaling with terawatt laser systems. New J. Phys. 2013, 15, 075002. [CrossRef]

15. Gorodetsky, A.; Koulouklidis, A.D.; Massaouti, M.; Tzortzakis, S. Physics of the conical broadband terahertz emission from two-color laser-induced plasma filaments. Phys. Rev. A 2014, 89, 033838. [CrossRef]

16. Andreeva, V.; Kosareva, O.; Panov, N.; Shipilo, D.; Solyankin, P.; Esaulkov, M.; de Alaiza Martínez, P.G.; Shkurinov, A.; Makarov, V.; Bergé, L.; et al. Ultrabroad terahertz spectrum generation from an air-based filament plasma. Phys. Rev. Lett. 2016, 116, 063902. [CrossRef]

17. Fedorov, V.Y.; Tzortzakis, S. Extreme THz fields from two-color filamentation of midinfrared laser pulses. Phys. Rev. A 2018, 97, 063842. [CrossRef]

18. You, Y.; Oh, T.; Kim, K. Off-axis phase-matched terahertz emission from two-color laser-induced plasma filaments. Phys. Rev. Lett. 2012, 109, 183902. [CrossRef]

19. Borodin, A.V.; Esaulkov, M.N.; Kuritsyn, I.I.; Kotelnikov, I.A.; Shkurinov, A.P. On the role of photoionization in generation of terahertz radiation in the plasma of optical breakdown. JOSA B 2012, 29, 1911-1919. [CrossRef]

20. Blank, V.; Thomson, M.; Roskos, H. Spatio-spectral characteristics of ultra-broadband THz emission from two-colour photoexcited gas plasmas and their impact for nonlinear spectroscopy. New J. Phys. 2013, 15, 075023. [CrossRef]

21. Rasmussen, M.; Jepsen, P.; Zhou, B. Accurately frequency-resolved air-plasma THz beam profile from knifeedge assisted waveform measurements. In Proceedings of the 2021 46th International Conference on Infrared, Millimeter and Terahertz Waves (IRMMW-THz), Chengdu, China, 16-17 August 2021.

22. Sørensen, C.B.; Guiramand, L.; Degert, J.; Tondusson, M.; Skovsen, E.; Freysz, E.; Abraham, E. Conical versus Gaussian terahertz emission from two-color laser-induced air plasma filaments. Opt. Lett. 2020, 45, 2132-2135. [CrossRef]

23. Ushakov, A.; Chizhov, P.; Andreeva, V.; Panov, N.; Shipilo, D.; Matoba, M.; Nemoto, N.; Kanda, N.; Konishi, K.; Bukin, V.; et al Ring and unimodal angular-frequency distribution of THz emission from two-color femtosecond plasma spark. Opt. Express 2018, 26, 18202-18213. [CrossRef]

24. Zhong, H.; Karpowicz, N.; Zhang, X.C. Terahertz emission profile from laser-induced air plasma. Appl. Phys. Lett. 2006, 88, 261103. [CrossRef]

25. Ushakov, A.; Chizhov, P.; Bukin, V.; Shipilo, D.; Panov, N.; Kosareva, O.; Garnov, S. Multiple Filamentation Effects on THz Radiation Pattern from Laser Plasma in Air. Photonics 2021, 8, 4. [CrossRef]

26. Li, H.; Zhang, Y.; Sun, W.; Wang, X.; Feng, S.; Ye, J.; Zhang, Y. Contribution of the optical rectification in terahertz radiation driven by two-color laser induced plasma. Opt. Express 2020, 28, 4810-4816. [CrossRef]

27. Smirnov, S.V.; Kulya, M.S.; Tcypkin, A.N.; Putilin, S.E.; Bespalov, V.G. Detection of the polarization spatial distribution of THz radiation generated by two-color laser filamentation. Nanosyst.-Phys. Chem. Math. 2017, 8, 613-619. [CrossRef]

28. Hah, J.; Jiang, W.; He, Z.; Nees, J.; Hou, B.; Thomas, A.; Krushelnick, K. Enhancement of THz generation by feedback-optimized wavefront manipulation. Opt. Express 2017, 25, 17271-17279. [CrossRef]

29. Kolesik, M.; Moloney, J.V. Nonlinear optical pulse propagation simulation: From Maxwell's to unidirectional equations. Phys. Rev. E 2004, 70, 036604. [CrossRef]

30. Ho, I.C.; Guo, X.; Zhang, X.C. Design and performance of reflective terahertz air-biased-coherent-detection for time-domain spectroscopy. Opt. Express 2010, 18, 2872-2883. [CrossRef]

31. Dai, J.; Clough, B.; Ho, I.C.; Lu, X.; Liu, J.; Zhang, X.C. Recent progresses in terahertz wave air photonics. IEEE Trans. Terahertz Sci. Technol. 2011, 1, 274-281. [CrossRef]

32. Xie, J.; Fan, W.H.; Chen, X. Systematic experimental study on a highly efficient terahertz source based on two-color laser-induced air plasma. Laser Phys. 2016, 26, 055002. [CrossRef]

33. Koulouklidis, A.; Fedorov, V.Y.; Tzortzakis, S. Spectral bandwidth scaling laws and reconstruction of THz wave packets generated from two-color laser plasma filaments. Phys. Rev. A 2016, 93, 033844. [CrossRef] 
34. Rodriguez, G.; Dakovski, G.L. Scaling behavior of ultrafast two-color terahertz generation in plasma gas targets: Energy and pressure dependence. Opt. Express 2010, 18, 15130-15143. [CrossRef] [PubMed]

35. Zhao, J.; Chu, W.; Wang, Z.; Peng, Y.; Gong, C.; Lin, L.; Zhu, Y.; Liu, W.; Cheng, Y.; Zhuang, S.; et al. Strong spatial confinement of terahertz wave inside femtosecond laser filament. ACS Photonics 2016, 3, 2338-2343. [CrossRef]

36. Chen, Y.; Marceau, C.; Génier, S.; Théberge, F.; Châteauneuf, M.; Dubois, J.; Chin, S.L. Elliptically polarized Terahertz emission through four-wave mixing in a two-color filament in air. Opt. Commun. 2009, 282, 4283-4287. [CrossRef]

37. Akhmedzhanov, R.; Ilyakov, I.; Mironov, V.; Suvorov, E.; Fadeev, D.; Shishkin, B. Plasma mechanisms of pulsed terahertz radiation generation. Radiophys. Quantum Electron. 2009, 52, 482-493. [CrossRef]

38. Wang, T.J.; Marceau, C.; Chen, Y.; Yuan, S.; Théberge, F.; Châteauneuf, M.; Dubois, J.; Chin, S.L. Terahertz emission from a dc-biased two-color femtosecond laser-induced filament in air. Appl. Phys. Lett. 2010, 96, 211113. [CrossRef]

39. Zhang, X.C.; Xu, J. Introduction to THz Wave Photonics; Springer: New York, NY, USA, 2010; Volume 29.

40. Dai, J.; Xie, X.; Zhang, X.C. Detection of broadband terahertz waves with a laser-induced plasma in gases. Phys. Rev. Lett. 2006, 97, 103903. [CrossRef]

41. Lu, Z.; Campbell, P.; Zhang, X.C. Free-space electro-optic sampling with a high-repetition-rate regenerative amplified laser. Appl. Phys. Lett. 1997, 71, 593-595. [CrossRef]

42. Ushakov, A.; Panov, N.; Chizhov, P.; Shipilo, D.; Bukin, V.; Savel'ev, A.; Garnov, S.; Kosareva, O. Waveform, spectrum, and energy of backward terahertz emission from two-color femtosecond laser induced microplasma. Appl. Phys. Lett. 2019, 114, 081102. [CrossRef]

43. Shipilo, D.; Nikolaeva, I.; Pushkarev, D.; Rizaev, G.; Mokrousova, D.; Koribut, A.; Grudtsyn, Y.; Panov, N.A.; Seleznev, L.; Liu, W.; et al. Balance of emission from THz sources in DC-biased and unbiased filaments in air. Opt. Express 2021, 29, 40687-40698. [CrossRef]

44. Baxter, J.B.; Guglietta, G.W. Terahertz spectroscopy. Anal. Chem. 2011, 83, 4342-4368. [CrossRef]

45. Wu, Q.; Zhang, X.C. Design and characterization of traveling-wave electrooptic terahertz sensors. IEEE J. Sel. Top. Quantum Electron. 1996, 2, 693-700.

46. Zhong, H.; Redo, A.; Chen, Y.; Zhang, X.C. THz wave standoff detection of explosive materials. In Proceedings of the Terahertz for Military and Security Applications IV, Orlando, FL, USA, 19 May 2006; SPIE, the International Society for Optics and Photonics: Bellingham, WA, USA, 2006; Volume 6212, p. 62120L.

47. Usami, M.; Yamashita, M.; Fukushima, K.; Otani, C.; Kawase, K. Terahertz wideband spectroscopic imaging based on twodimensional electro-optic sampling technique. Appl. Phys. Lett. 2005, 86, 141109. [CrossRef]

48. Mankova, A.; Borodin, A.; Kargovsky, A.; Brandt, N.; Luo, Q.; Sakodynskaya, I.; Wang, K.; Zhao, H.; Chikishev, A.Y.; Shkurinov, A.; et al. Terahertz time-domain and FTIR spectroscopic study of interaction of $\alpha$-chymotrypsin and protonated tris with 18-crown-6. Chem. Phys. Lett. 2013, 560, 55-59. [CrossRef]

49. Bergé, L.; Kaltenecker, K.; Engelbrecht, S.; Nguyen, A.; Skupin, S.; Merlat, L.; Fischer, B.; Zhou, B.; Thiele, I.; Jepsen, P.U. Terahertz spectroscopy from air plasmas created by two-color femtosecond laser pulses: The ALTESSE project. EPL (Europhys. Lett.) 2019, 126, 24001. [CrossRef]

50. THz Band Pass Filters. Available online: www.tydexoptics.com/products/thz_assemblies/thz_band_pass_filter/ (accessed on 22 July 2021).

51. Nikolaeva, I.; Shipilo, D.; Pushkarev, D.; Rizaev, G.; Mokrousova, D.; Koribut, A.; Grudtsyn, Y.; Panov, N.; Seleznev, L.; Liu, W.; et al. Flat-top THz directional diagram of a DC-biased filament. Opt. Lett. 2021, 46, 5497-5500. [CrossRef]

52. Hansen, R.C. Phased Array Antennas; John Wiley \& Sons: Hoboken, NJ, USA, 2009; Volume 213.

53. D'Amico, C.; Houard, A.; Franco, M.; Prade, B.; Mysyrowicz, A.; Couairon, A.; Tikhonchuk, V. Conical forward THz emission from femtosecond-laser-beam filamentation in air. Phys. Rev. Lett. 2007, 98, 235002. [CrossRef]

54. D'Amico, C.; Houard, A.; Akturk, S.; Liu, Y.; Le Bloas, J.; Franco, M.; Prade, B.; Couairon, A.; Tikhonchuk, V.; Mysyrowicz, A Forward THz radiation emission by femtosecond filamentation in gases: Theory and experiment. New J. Phys. 2008, $10,013015$.

55. Panov, N.A.; Kosareva, O.G.; Andreeva, V.; Savel'ev, A.; Uryupina, D.S.; Volkov, R.V.; Makarov, V.; Shkurinov, A.P. Angular distribution of the terahertz radiation intensity from the plasma channel of a femtosecond filament. JETP Lett. 2011, 93, 638. [CrossRef]

56. Orlova, E.; Hovenier, J.; Klaassen, T.; Kašalynas, I.; Adam, A.; Gao, J.; Klapwijk, T.; Williams, B.; Kumar, S.; Hu, Q.; et al. Antenna model for wire lasers. Phys. Rev. Lett. 2006, 96, 173904. [CrossRef] [PubMed]

57. Klarskov, P.; Strikwerda, A.C.; Iwaszczuk, K.; Jepsen, P.U. Experimental three-dimensional beam profiling and modeling of a terahertz beam generated from a two-color air plasma. New J. Phys. 2013, 15, 075012. [CrossRef]

58. Panov, N.; Andreeva, V.; Kosareva, O.; Shkurinov, A.; Makarov, V.; Bergé, L.; Chin, S. Directionality of terahertz radiation emitted from an array of femtosecond filaments in gases. Laser Phys. Lett. 2014, 11, 125401. [CrossRef]

59. Gallot, G.; Zhang, J.; McGowan, R.; Jeon, T.I.; Grischkowsky, D. Measurements of the THz absorption and dispersion of ZnTe and their relevance to the electro-optic detection of THz radiation. Appl. Phys. Lett. 1999, 74, 3450-3452. [CrossRef]

60. Varga, P.; Török, P. Focusing of electromagnetic waves by paraboloid mirrors. I. Theory. J. Opt. Soc. Am. A 2000, 17, 2081-2089. [CrossRef]

61. Ahi, K. Mathematical modeling of THz point spread function and simulation of THz imaging systems. IEEE Trans. Terahertz Sci. Technol. 2017, 7, 747-754. [CrossRef] 